\title{
PHYSICS OF LASER-INDUCED PLASMA STREAMS UNDER IRRADIATION OF METALS WITH NANOSECOND LASER RADIATION PULSES AT ATMOSPHERIC PRESSURE
}

\author{
K. V. Kozadaev
}

UDC 535.35

We have investigated the physics of the processes of incipient formation, development, and breakdown of vaporplasma streams formed under the action of high-power $\left(10^{8}-10^{9} \mathrm{~W} / \mathrm{cm}^{2}\right)$ nanosecond laser pulses on massive metal targets in air. The main theoretical models of the process of laser erosion of metals by nanosecond pulses have been considered. A comparison has been made between the results of experimental and theoretical investigations of the laser erosion processes.

Keywords: laser erosion of metals, nanosecond laser pulse, erosion laser torch, hydrodynamic model, condensation, metal nanoparticles.

Introduction. The problems of plasma physics have been attracting close attention from researchers practically ever since high-power optical quantum generators were created. The large body of results of experimental and theoretical investigations amassed over the past 50 years formed the basis for many thousands of scientific publications. A number of monographs [1-10] generalizing the existing notions on the physics of interaction of laser radiation with various materials have been published, and now these works can rightly be considered as chrestomathies. However, in spite of the enormous body of available information, these problems are still of great practical interest, which is evidenced by new surveys and theoretical works [11-18].

In the overwhelming majority of existing theoretical publications modeling the regimes of irradiation of metals with nanosecond laser pulses, the vacuum approximation is used, since the presence of foreign gases complicates considerably the mathematical description of the above process [16-18]. On the other hand, recent works [19-24], because of their narrow practical purposefulness, are characterized by widely disconnected topics, and, therefore, it is very difficult to reconstruct on their basis the integral physical picture of the phenomenon under investigation. However, the results of a number of works [25-28] show that the processes of plasma initiation under the action of high-power nanosecond laser pulses on metals in air can serve as the basis for developing effective technologies of laser treatment, including those aimed at bulk [29-31] and surface [32] modification of functional materials by metal nanostructures.

The present paper is devoted to the investigation of the basic aspects of the processes of initiation, development, and breakdown of vapor-plasma streams formed under the action of high-power nanosecond laser pulses on massive metal targets in air at atmospheric pressure.

Processes of Laser Radiation Energy Dissipation in a Metal Lattice. According to the classical notions, when optical radiation is incident on the surface of a metal, in the initial stage in a thin $(1 \mu \mathrm{m})$ near-surface layer its interaction with conduction electrons occurs, as a result of which a portion of the radiation energy is reflected, and the remaining portion is expended in increasing the electron gas temperature and, subsequently, in heating the atomic lattice of the material [1-3]. Hereinafter all considerations are given for the case without air breakdown by laser radiation. The effect of low-threshold breakdown of air and its influence on the processes of laser erosion of materials were considered in detail in [33-35]. Electromagnetic radiation energy transfer deep into the metal is possible due to the following three main types of heat conductivity: phonon, electron, and radiant heat conductivities. It is customary to assume that the phonon heat conductivity plays a significant role at temperatures below $100 \mathrm{~K}$ and, therefore, it is not taken into account in investigations. In the temperature range of the lattice from hundreds to thousands of kelvins the energy transfer mechanism is practically fully determined by the processes of electron heat conductivity. Finally, as the temperature is increased from units to dozens of thousands of kelvins, the

A. N. Sevchenko Research Institute of Applied Physical Problems, Belarusian State University, 7 Kurchatov Str., 220108, Minsk, Belarus; email: kozadaeff@mail.ru. Translated from Inzhenerno-Fizicheskii Zhurnal, Vol. 87, No. 3, pp. 682-693, May-June, 2014. Original article submitted October 4, 2013. 

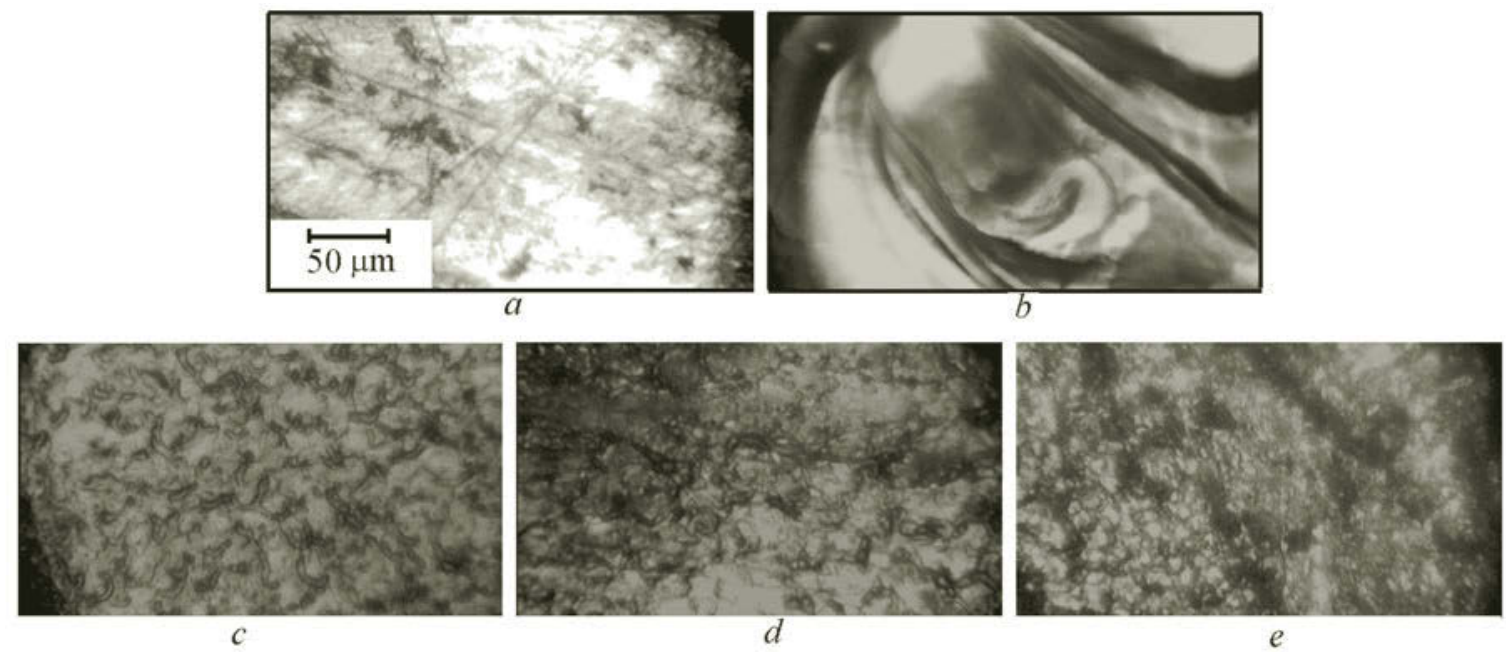

Fig. 1. Surface relief of the nickel target before (a) and after (b) its irradiation with a laser radiation of moderate power density and surface reliefs of the nickel (c), zinc (d), and lead (e) targets after their irradiation with a 100 -ns pulse of intensity $10^{9} \mathrm{~W} / \mathrm{cm}^{2}$.

radiant heat conductivity dominates. However, since at such temperatures under normal conditions the existence of a metal in the form of a solid (or even a liquid) is unlikely, the corresponding processes belong to the category of fast ones, as a rule [1].

The processes of laser energy dissipation in metals at a laser pulse length $t \sim 10 \mathrm{~ns}$ differ qualitatively from both longer pulses $(t>1 \mu \mathrm{s})[1-10,14,36]$ and ultrashort ones $(t<100 \mathrm{ps})[11-13,16,23,37-40]$. This is due to the value of the characteristic time of the lattice temperature change determined by the rate of energy exchange between conduction electrons and lattice sites: theoretical estimations give a value of $\sim 10^{-10} \mathrm{~s}[1]$. However, for real metals this parameter can be an order of magnitude higher than the model value. Such a situation leads to the fact that the characteristic time scale of the relaxation process of the electron gas excitation energy in the atomic lattice of the metal turns out to be comparable to the duration of the leading edge of the radiation pulse acting on the metal (units of nanoseconds), i.e., the rate of excitation transfer from the electron gas to the atomic lattice of the metal approaches the rate of input to the metal of radiant energy causing electron gas excitation. In this case, there is a sharp decrease in the value of the electron heat conductivity as the main transfer mechanism of the energy absorbed by the conduction electrons, deep into the metal. Moreover, because of the sharp decrease in the electrical conductivity of the metal caused by its fast heating to a temperature of $\sim 1000-1500 \mathrm{~K}$ (characteristic of the action of nanosecond pulses with a power density $W \sim 10^{8}-10^{9} \mathrm{~W} / \mathrm{cm}^{2}$ [1], the penetration depth of radiant energy into the metal increases many times [2]. This fact causes one to reconsider the estimate of the thickness of the near-surface layer of the metal, in which the interaction of laser radiation with conduction electrons occurs, made in [1-3]: instead of $1 \mu \mathrm{m}$ this thickness can be equal to 5-10 $\mu \mathrm{m}$ under a fairly powerful laser irradiation [27]. It should also be noted that under a high-power nanosecond laser irradiation of the metal the coefficient of reflection of the radiation by the metal surface is not constant: beginning from a certain threshold value of the laser radiation power (by different estimates, $W<100 \mathrm{MW} / \mathrm{cm}^{2}[1,41,42]$ ) the initially high value of the reflection coefficient (in the optical range of 0.7-0.99, depending on the type of metal [43]) in the process of action of this radiation on the metal can decrease many times, now and then bringing the integral dose of absorbed energy to $50 \%$ of the incident radiation dose [1]. This phenomenon causes one to reconsider the laser radiation dose initially absorbed by the metal, assuming it to be larger.

The simultaneous influence of the above effects on the erosion processes leads to the fact that already at $W>10^{8} \mathrm{~W} / \mathrm{cm}^{2}$ the damage of metals by nanosecond laser pulses is difficult to describe from the viewpoint of the traditional thermal damage (quasi-stationary ablation) model $[16,44]$. The basis for the description of the process of laser radiation erosion of metals in the thermal model approximation is the simultaneous propagation of the heating, melting, and evaporation fronts deep into the target material, i.e., in the laser irradiation zone of the metal three aggregate states of the metal are present simultaneously: solid, liquid, and gas (in the case of exposure to a rather intense radiation, weakly ionized plasma can be added to this list of states) [1-3, 16]. For longer pulses $(t>1 \mu \mathrm{s})$, such a model approximation permits obtaining results close to real ones [36]. However, as the leading edge of the laser pulse is decreased to $1-10 \mathrm{~ns}$, already at $W \sim 10^{8}-10^{9} \mathrm{~W} / \mathrm{cm}^{2}$ the sharp interphase 
boundaries between the above aggregate states of the metal diffuse into a macroscopic transition layer, whose thickness is determined by the penetration depth of the light pulse $(5-10 \mu \mathrm{m})$ [27]. In this layer, actually the whole of the absorbed laser pulse energy dissipates, and the excess of imparted energy makes it similar to an explosive - the macrolayer transforms rather rapidly (as far as the matter inertia allows) to a rapidly expanding superheated vapor (according to the estimates of $[45,46]$, the initial velocity of expansion of such vapor reaches $10-20 \mathrm{~km} / \mathrm{s}$ ) forming subsequently an erosion torch. This active gas-dynamic process imparts a strong mechanical impulse of recoil pressure initiating an acoustic shock wave propagating deep into the target material [1,2,22].

The described mechanism of laser erosion of metals is called in the literature the "hydrodynamic" mechanism [1], which is due to the use of the hydrodynamic approximation in describing the process of outflow of vapors of the target material. It should be noted, however, that the hydrodynamic mechanism of damage of metals was predicted theoretically for nanosecond laser pulses with a much higher power density $\left(W>10^{11} \mathrm{~W} / \mathrm{cm}^{2}\right)[1]$ and, therefore, we have to state that the real boundary of the range of radiation power density necessary for activating the above mechanism is several orders of magnitude lower. The fundamental difficulty of a theoretical description of the process of laser erosion of metals in the hydrodynamic approach is the lack of reliable data on the coefficient of absorption of laser radiation by the dense nonstationary plasma formed by detonation of the macrolayer. As a result of this, it has been impossible so far to obtain satisfactory agreement between theoretical and experimental data, and researchers are satisfied with describing only individual processes proceeding in the near-surface region of metals irradiated under the conditions being considered [17, 18]. An additional difficulty thereby is taking into account the influence of external atmospheric gases. In this case, the discrepancy between theoretical and experimental estimates is even wider [16].

As mentioned above, the rapid expansion of the vapor-gas formation towards the environment by the mechanical momentum conservation law leads to the formation of an acoustic recoil wave propagating deep into the material of the metal. The shock acoustic wave imparts much energy to the laser target, which may leads to additional melting and evaporation of the thin near-surface layer of the metal. However, since the propagation velocity of the acoustic wave is lower than the characteristic velocity of the electron heat conductivity, its mechanical energy dissipates according to the thermal model and, therefore, because a relatively large volume of the target material is involved in the process, the influence of this effect on the gas dynamics of the erosion laser torch (ELT) and the formation of the end surface relief of the target is weak, as a rule $[1,2]$. According to the estimates of [22], the efficiency of ablation loading (the portion of the laser energy contained in the shock acoustic wave) of the metal is only a few percent for a power density of nanosecond pulses of $10^{12}-10^{14} \mathrm{~W} / \mathrm{cm}^{2}$ (for a less intense radiation the value of this parameter will be even lower).

It should be noted that the mechanical pressure momentum imparted to the target by the acoustic recoil wave is a convenient parameter for quantitative measurements characterizing the process of laser irradiation of the metal. If we take into account that the value of the recoil pressure momentum imparted to the target on the whole characterizes fairly exactly the initial kinetic energy of the vapor-gas formation, then, measuring this parameter, it is possible to characterize quantitatively the efficiency of laser irradiation of the metal (at least from the point of view of erosion processes). In [2], it was shown that the value of such a pressure momentum under irradiation of a metal with high-power nanosecond laser pulses $\left(W \sim 10^{9} \mathrm{~W} / \mathrm{cm}^{2}\right)$ depends rather weakly on the radiation wavelength (the other radiation characteristics being comparable): in the optical range of wavelengths of $0.3-1.0 \mu \mathrm{m}$, the value of the recoil pressure momentum changes by no more than $10 \%$; moreover, as the laser radiation wavelength is increased by an order of magnitude ( $\lambda=10.6 \mu \mathrm{m}$ for the $\mathrm{CO}_{2}$ laser), the value of the recoil pressure momentum decreases by a factor of only a little more than 2 . On this basis, we can state that the influence of the factor of laser radiation wavelength on the efficiency of laser erosion of metals by nanosecond pulses is not significant, more important parameters being the steepness of the leading edge of the radiation pulse and its power density.

Initiating the above processes in the metal by nanosecond laser pulses leads finally to a characteristic change in its surface relief (Fig. 1c-e) differing widely from the surface relief of this metal irradiated with both long laser radiation pulses [27] and ultrashort ones [20, 23, 40]. From Fig. 1 it is seen that with the thermal mechanism of damage of the metal (characteristic of long laser radiation pulses) the mirror relief of its surface is formed by solidification of a fairly deep layer of the metal melt (Fig. 1b). The hydrodynamic mechanism of erosion of the metal leads to the appearance on its surface of a characteristic microchannel structure with rather sharp contours of its edges (the estimated sizes of the roundings-off and bulges of the channel walls are a few micrometers), which points to the practically complete absence of the liquid phase from the metal (Fig. 1c-e) [27]. The simultaneous influence of several factors (the initial relief of the target, the nonideality of its material, the inhomogeneity of the incident radiation, the boundary conditions of the gas dynamics, etc.) on the processes proceeding in the macrolayer of the metal determine the unique pattern of microchannel structures (for smooth and highly 

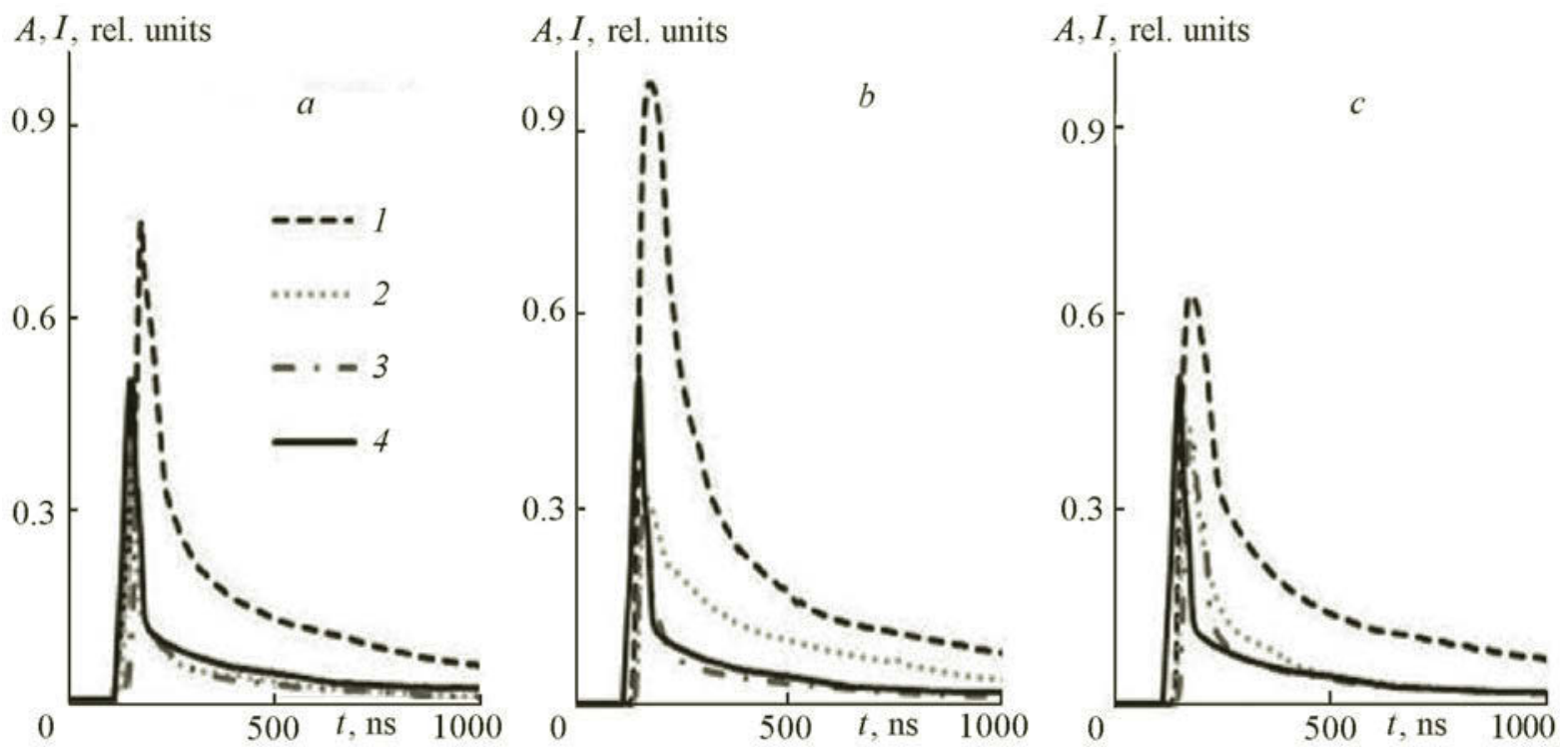

Fig. 2. Integrated luminescence dynamics of the zinc (a), lead (b), and nickel (c) ELTs under the action on them of 20-ns laser pulses with $W=10^{9}(1), 3 \cdot 10^{8}$ (3), and $10^{8}$ $\mathrm{W} / \mathrm{cm}^{2}$ (3): 4) time dependence of the laser radiation pulse intensity.
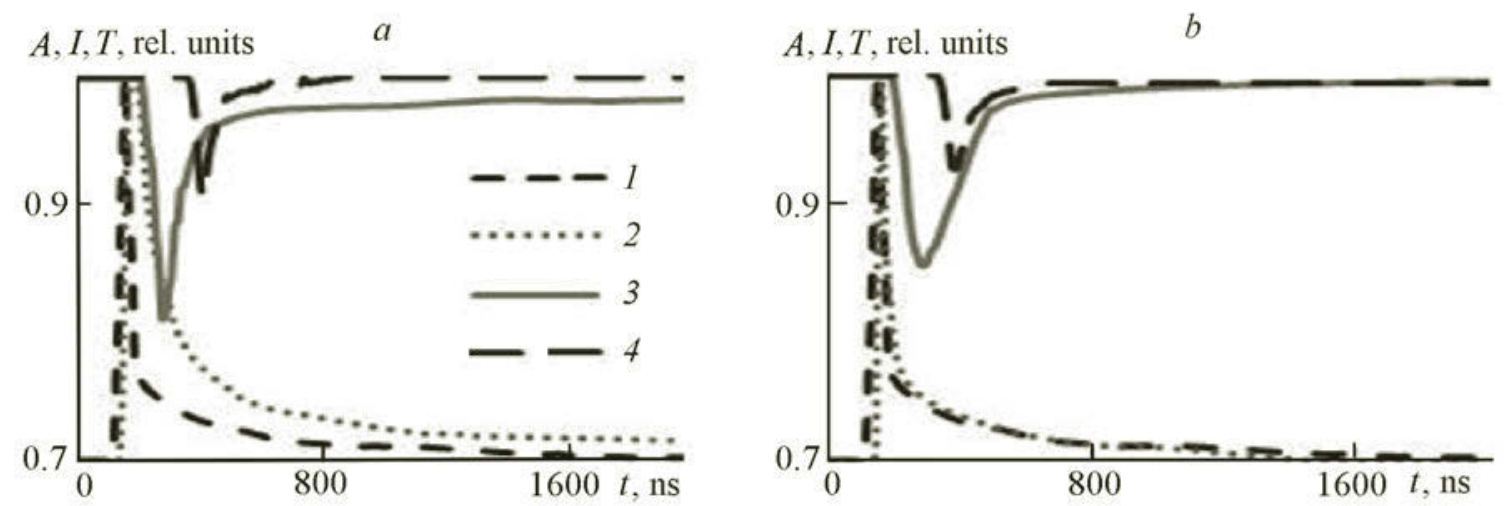

Fig. 3. Time dependences of the acting 20-ns laser pulse intensity (1), the integrated luminosity of the ELT (2), and the transmissivity of the ELT for the probing radiation $(3,4)$ at a distance from the lead target surface $h=1 \mathrm{~mm}(3)$ and $h=2 \mathrm{~mm}(4)$ : a) $W=$ $10^{9} \mathrm{~W} / \mathrm{cm}^{2}$; b) $10^{8}$.

homogeneous targets a pattern in the form of an aggregate of microcraters is possible) [20]. With increasing power density of nanosecond pulses a certain smoothness of the microchannel structures takes place due to the back action of the nearsurface plasma on the target, which is possible owing to the shielding from the radiation by the laser plasma and the complex character of its interaction with ambient gases (in vacuum this effect is practically not pronounced). In the case of laser erosion of metals by ultrashort pulses with a comparable radiant energy density $\left(\sim 10 \mathrm{~J} / \mathrm{cm}^{2}\right)$, because of the shortening of acting pulses by several orders of magnitude, their power density increases, which increases considerably the probability of optical breakdown of air $[34,35]$ and, therefore, the efficiency of laser irradiation of the metal sharply decreases due to the shielding of the target by the breakdown plasma. Low-energy ultrashort pulses with an energy density lower than $1 \mathrm{~J} / \mathrm{cm}^{2}$, as a rule, do not cause developed erosion of the target — the estimated values of the ablated layer thickness are of a few dozens of nanometers and, therefore, significant changes in the metal relief take place upon its repeated (hundreds and thousands times) irradiation [47]. 

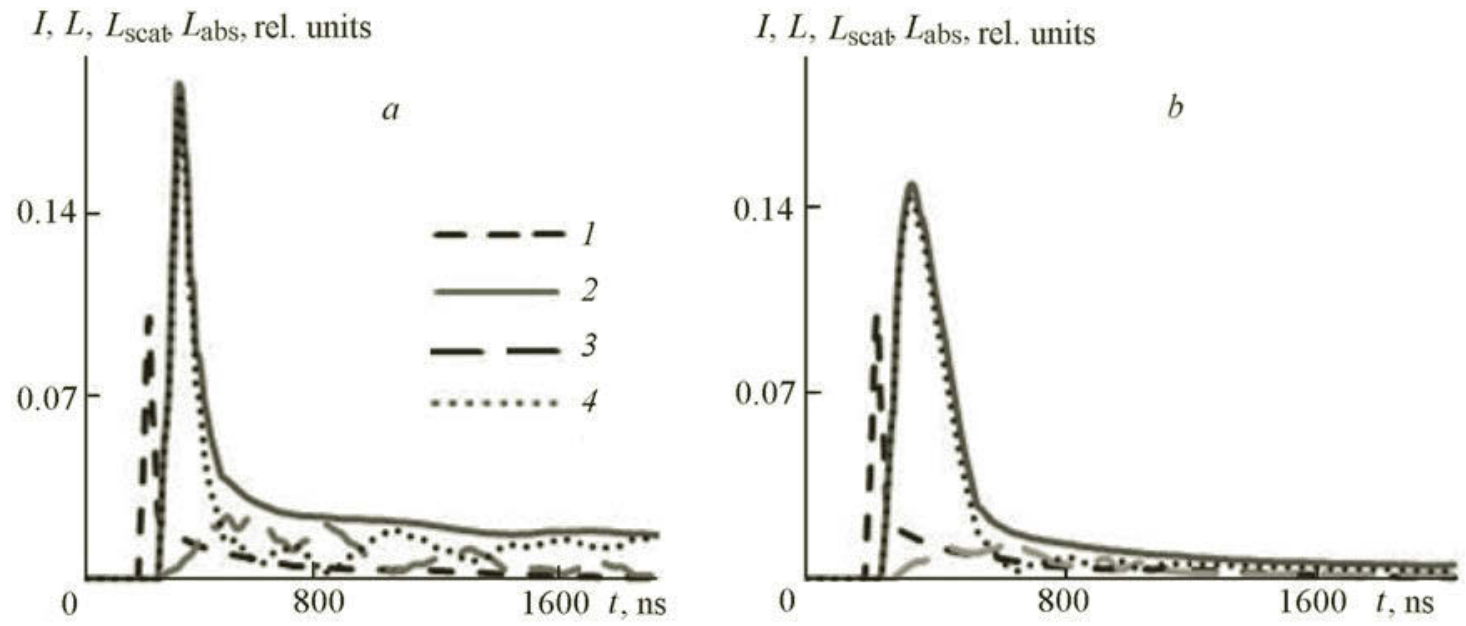

Fig. 4. Time dependences of the acting 20-ns laser pulse intensity (1), the total probing radiation loss in the ELT (2), and the probing radiation loss in the ELT due to the scattering (3), the probing radiation loss in the ELT due to its absorption in the plasma (4) at a distance from the lead target surface $h=1 \mathrm{~mm}$ : a) $W=10^{9} \mathrm{~W} / \mathrm{cm}^{2}$; b) $10^{8}$.

Initiation of a Metal Vapor-Plasma Formation at Atmospheric Pressure. As noted in the previous section, irradiation of a metal with high-power nanosecond laser pulses leads to the formation in it of a macrolayer, whose thickness is determined by the penetration depth of the radiation and which has a considerable amount of excess energy. This leads to the fact that shortly after the beginning of the laser irradiation (with a delay of 5-10 ns for the 20-nanosecond pulse [45] and 30-40 ns for the 100-nanosecond pulse [46]), in the near-surface region of the target there appears a bright luminous vaporplasma formation expanding rapidly in the direction of the environment while interacting with the acting laser pulse [48]. Since the processes of vapor outflow into a vacuum and into a gas atmosphere differ radically, the spatial, energy, spectral, and other characteristics of erosion torches will differ considerably even under comparable irradiation conditions [15, 16, 49, 50].

The main body of existing theoretical data on the topic under consideration pertains to the processes of expansion of laser-induced vapor-plasma formations in vacuum (moreover, a one-dimensional case is often considered). The gas dynamics of the laser torch is determined thereby by the regime of evaporation of the substance from the target surface, the interaction of the incident radiation with the vapor-plasma formation, and the influence of the condensation processes of the vaporplasma cloud [1,16-18]. For the main regime of evaporation, one considers the approximation of the laser erosion thermal model, in which the flow velocity of the gas phase into the torch is determined by the quasi-steady motion of the evaporation front, although in some works $[16,44]$ the authors note an essentially nonstationary character of initiation of a vapor-plasma formation under the action of high-power nanosecond pulses on metals. Then the gas phase of the target material consisting of vapors and weakly ionized plasma begins to flow into a vacuum (in $[1,2,16]$, the expansion regimes of supersaturated vapor, as well as the regimes of adiabatic and isothermal expansion of the gas, are described), continuing to interact with the incident radiation. According to [51], at temperatures below $10^{-4} \mathrm{~K}$ the optical radiation absorption in the gas is determined by the following two processes: photoelectric absorption of radiation by excited atoms and retarding radiation absorption by electrons in the field of ions and neutral atoms. According to the estimates of [1], the contribution of the retarding radiation absorption by electrons in the field of neutral atoms in the case of initiation of metal erosion by high-power laser pulses is insignificant and, therefore, under the conditions of thermodynamic equilibrium for the overall coefficient of radiation absorption the Kramers-Unsold relation holds [51], and for $W=10^{8}$ and $10^{9} \mathrm{~W} / \mathrm{cm}^{2}$ the process of appreciable absorption of the radiant energy by the gas flow (the optical plasma density is $\sim 1$ ) begins, respectively, $10^{-5}$ and $10^{-7} \mathrm{~s}$ after the onset of evaporation [1].

Experimental studies of the gas-dynamic processes taking place in the case of laser erosion of metals by nanosecond pulses in the presence of rarefied gases [24, 49] and at atmospheric pressure [25, 26, 45, 46, 48, 52] showed a qualitative difference between the processes of initiation of a vapor-plasma formation in air and its interaction with the incident radiation as compared to the case of initiation of a vapor-plasma formation in vacuum [53-56]. In [45, 46, 48], it was shown 



Fig. 5. SEM image of precipitated gold (a) and silver (b) nanoparticles, TEM image of polyvinyl alcohol film containing silver nanoparticles (c), and SEM image of the silver island nanostructures on the silicon surface $(\mathrm{d})$.

experimentally that the luminosity dynamics of the vapor-plasma formation torch under the action of laser pulses of length 20 and $100 \mathrm{~ns}$ (the length of the leading edge of pulses is 5 and $30 \mathrm{~ns}$, respectively) and intensity $W \sim 10^{8} \mathrm{~W} / \mathrm{cm}^{2}$ on metals $\mathrm{Zn}, \mathrm{Pb}, \mathrm{Ni}, \mathrm{Cu}, \mathrm{Ag}$, and Au replicates practically completely the time form of the laser pulse with a short delay $(\sim 50-60 \%$ of the pulse length), which points to the absence of a significant interaction between the erosion torch and the laser radiation. This statement is confirmed additionally by the data on auxiliary radiation probing of erosion torches at heights $h=1$ and $h=$ $2 \mathrm{~mm}$ from the target surface: the vapor-plasma formation remains practically transparent to the probing radiation during its lifetime (a certain deviation is demonstrated only by torches of fusible metals $\mathrm{Zn}$ and $\mathrm{Pb}$ for pulses of length $100 \mathrm{~ns}$ ). As the power density of acting nanosecond pulses is increased to $10^{9} \mathrm{~W} / \mathrm{cm}^{2}$, there is a sharp increase in the luminescent intensity of the vapor-plasma formation, as well a considerable pulling of its trailing edge (Fig. 2) [48]. A simultaneous decrease in the torch transparency to the probing radiation is observed (Fig. 3) [45].

The above facts show that an appreciable interaction between the metal erosion torch and the trailing edge of nanosecond pulses (radiant energy absorption) begins when their power densities approach $10^{9} \mathrm{~W} / \mathrm{cm}^{2}$ (it should be noted that for different metals this threshold value may be somewhat different, which is due to the difference in their thermal characteristics [43]), and noticeable radiation absorption by the torch plasma begins much earlier than the damage model predicts: 40-50 ns after the beginning of irradiation for 100-ns pulses and $10 \mathrm{~ns}$ for 20 -ns pulses instead of the calculated $100 \mathrm{~ns}$. Investigations $[45,46,48]$ showed that the maximum luminescence of erosion torches for $20-\mathrm{ns}$ pulses $\left(W=10^{9} \mathrm{~W} / \mathrm{cm}^{2}\right)$ is localized at a height of less than $1 \mathrm{~mm}$ from the target surface, whereas for 100-ns pulses of analogous intensity it is localized at a height of 1-2 mm, which is explained by the increased radiant energy density. This permits characterizing the spatial scales of the erosion torch at the beginning of their nonstationary formation in air at atmospheric pressure. The data on the probing of erosion torches at heights $h=1$ and $h=2 \mathrm{~mm}$ from the metal target surface permitted estimating the velocities of their propagation in the above time intervals: $4-15 \mathrm{~km} / \mathrm{s}$ for $20-\mathrm{ns}$ pulses [47] and 7-20 km/s for $100-\mathrm{ns}$ pulses [46] $\left(W=10^{9}\right.$ 

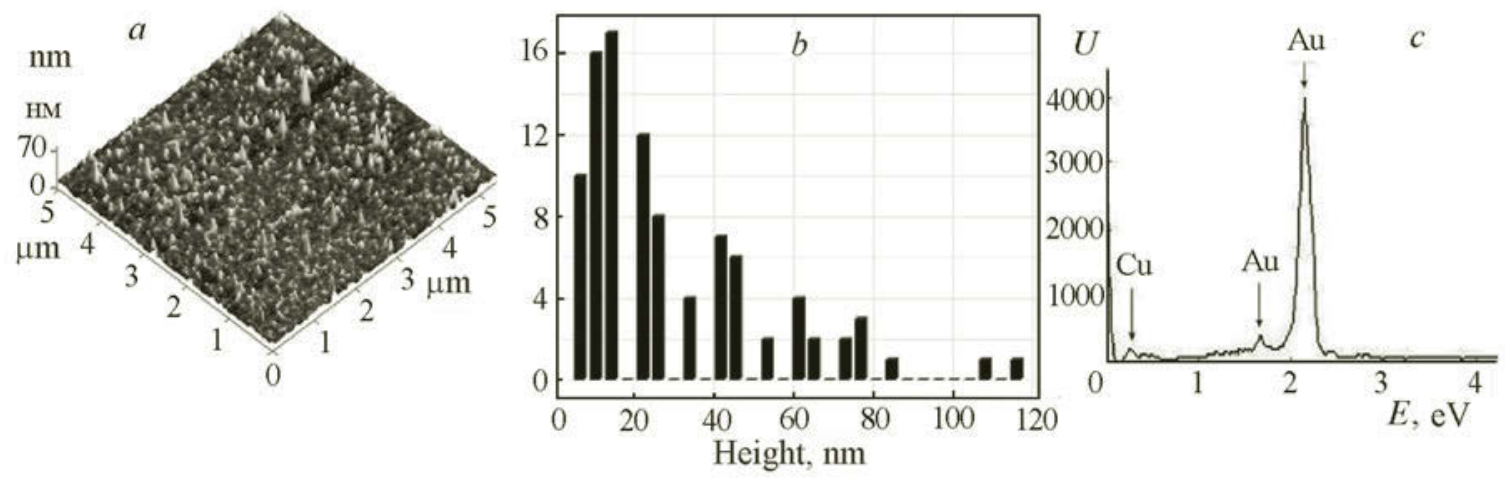

Fig. 6. AFM image of the silicon substrate surface with precipitated gold nanoparticles (a), height distribution of conic nanostructures, and characteristic radiation spectrum of a conic nanoobject excited by a narrow-focus beam (c).

$\mathrm{W} / \mathrm{cm}^{2}$ ) depending on the type of metal. The absence of a significant influence of the steepness of the leading edge of the pulse on the plasma formation velocity points to the fact that the above velocities are limiting for the described regime of laser irradiation of the metal, and the initial dynamics of the torch is only determined by the velocity of detonation of the macrolayer. Investigations of the spectral-time structure of erosion torches of metals $\mathrm{Zn}, \mathrm{Pb}$, and $\mathrm{Ni}$ in the case of their irradiation with 20-ns pulses $\left(W \sim 10^{8}-10^{9} \mathrm{~W} / \mathrm{cm}^{2}\right)$ showed [48] that the process of erosion torch luminescence can be subdivided into two stages - the formation of a continuous luminescence spectrum with a short delay ( $50 \%$ of the pulse length) from the beginning of irradiation and a slower (with a delay of $\sim 700 \mathrm{~ns}$ ) manifestation of the characteristic lines of the metal, and the major portion of continuum luminescence being spatially localized at a height of up to $1 \mathrm{~mm}$. Similar effects were observed in the case of laser erosion of metals by nanosecond pulses in a rarefied atmosphere at a pressure of $\sim 10 \mathrm{~Pa}$ $[24,29]$. Indeed, in the given case the spatial scale of the phenomena was much larger; for example, the longitudinal size of the region of continuum luminescence reached $5-10 \mathrm{~mm}$.

Condensation of the Erosion Laser Torch at Atmospheric Pressure. Upon termination of laser irradiation of the metal the erosion torch, because of its expansion, begins to cool down, which leads to a recombination of the free charge carriers in the plasma and an increase in its transparency and, subsequently, to the condensation of metal vapors [1]. The theoretical consideration of the condensation processes under such conditions pertains to problems of the kinetics of fast phase transitions of the first kind, which has up to now been little studied. The typical cooling rates of vapor upon expansion of a cloud of ablation products into a vacuum are $10^{10}-10^{11} \mathrm{~K} / \mathrm{s}$. Earlier the classical physics of phase transitions did not give as much consideration to the nonequilibrium condensation conditions [16]. To describe such processes theoretically, the theory of dynamic condensation of expanding vapor (Zel'dovich-Raizer theory) was proposed [51]. There are certain difficulties in describing the characteristic time scales of condensation processes in this approximation; however, the Zel'dovich-Raizer theory permits getting a graphic picture of the process physics and the size of clusters formed in vacuum [57]. According to this theory [51], the initial vapor expansion occurs along the Poisson adiabat, then the vapor cools down and goes to the saturation state (the Poisson adiabat intersects the saturation adiabat given by the Clapeyron-Clausius equation). From this moment the condensation process begins. In this process, three waves propagate sequentially (from the periphery to the center) through the vapor cloud: the saturation wave, the wave of "injection" of critical nuclei (the moment when the vapor supercooling is maximum), and the wave of "hardening," i.e., of final formation of clusters. For the typical parameters of the cloud of laser ablation products, the time interval from the moment irradiation begins to the completion of "hardening" is a few microseconds [16]. As a result, a cloud of nanoclusters of the target material with characteristic sizes of 5-10 nm appears $[16,57]$. Even in the absence of adjustable parameters, the Zel'dovich-Raizer theory permits obtaining good agreement with experiment; however, the additional account of the influence of the atmosphere of external gases and the real pressure and density profiles in the vapor cloud strongly complicates the description of the condensation processes in air [16].

For experimental studies of the dynamics of the processes proceeding in a metal erosion torch upon termination of laser irradiation in [45, 46], we used the method of transverse laser probing of such a torch with parallel division of the radiation extinction in the torch into an absorbed and a scattered components. As an example, let us consider the dynamics of 
the optical characteristics of a lead erosion torch under the action of nanosecond laser pulses with $W \sim 10^{8}$ and $W \sim 10^{9}$ $\mathrm{W} / \mathrm{cm}^{2}$ on the metal in air at atmospheric pressure. Probing was carried out at a height $h=1 \mathrm{~mm}$ from the metal surface (Fig. 4) [47]. From Fig. 4a it is seen that in the first 150 ns after the beginning of irradiation of the target practically all losses of the probing radiation in the torch are determined by the plasma absorption (the dissipation mechanisms of the radiant energy were considered in the previous section). Then in the structure of the probing radiation extinction there appears a scattered component that increases rapidly and reaches its maximum about $200 \mathrm{~ns}$ after the beginning of irradiation. Then this component decreases nonmonotonically, and about $1.5 \mu$ s after the beginning of irradiation, a stable prevalence of the processes of absorption over scattering at a low general level of probing radiation losses in the torch is observed. A qualitatively identical experimental pattern is demonstrated by the lead erosion torch under the action of 100-ns pulses [46]: differences only in the time scales of the processes are observed: the scattered component reaches its maximum $800 \mathrm{~ns}$ after the beginning of laser irradiation, and stable prevalence of absorption over scattering is reached only $3 \mu$ s after this irradiation.

The above experimental effects can be interpreted from the viewpoint of the Zel'dovich-Raizer theory as follows. At the first stage of ELT formation, its interaction with the trailing edge of the incident radiation pulse occurs, due to which the torch plasma increases considerably its density and temperature, which enables it to remain opaque to the probing radiation during hundreds of nanoseconds after the laser irradiation. Then a sharp decrease in the optical density of the torch followed by the appearance of a scattered component begins. This moment can correspond to the propagation of the vapor saturation wave at the probing height. In the cooled vapor-plasma formation, density inhomogeneities are formed, leading to an increase in its luminescence component. The fact that this component reaches its maximum points to the appearance of a large number of scattering centers, which is indicative of the propagation of the wave of "injection" of critical nuclei, whose sizes increase subsequently due to the decrease in the torch density, leading to an increase in its transparency. At the final stage, absorption begins to prevail over scattering again at a practically total transparency of the torch. This effect can be explained by the completion of the formation of the condensed phase of the target material, i.e., the propagation of the "hardening" wave at the probing height.

A cloud of the condensed phase of the metal can be observed in the near-surface zone of the target for 500-700 $\mu \mathrm{s}$ more (this is evidenced by the data of laser probing in a longer time interval) $[25,26]$, which points to its comparatively slow expansion. The characteristic particle size of the condensed phase of the target material is $20-140 \mathrm{~nm}$ depending on the type of metal and the laser excitation conditions (lower-melting metals have thereby comparatively larger effective diameters of particles than refractory metals), which exceeds 5-10 times their estimated sizes according to the Zel'dovich-Raizer theory for the case of vacuum [57]. The typical numerical values of concentrations of such particles in the near-surface region of the target are $10^{9}-10^{12} \mathrm{~cm}^{-3}$ for 20-ns pulses [26] and $10^{10}-10^{13} \mathrm{~cm}^{-3}$ for 100-ns pulses [27]. Experimental data point to the fact that the action of lower-power particles $\left(W \sim 10^{8} \mathrm{~W} / \mathrm{cm}^{2}\right)$ is more effective from the viewpoint of the condensed phase formation than for pulses with $W \sim 10^{9} \mathrm{~W} / \mathrm{cm}^{2}$ (the particle concentration decreases by an order of magnitude or more). This can be explained by the fact that as $W$ increases from $10^{8} \mathrm{~W} / \mathrm{cm}^{2}$ to $10^{9} \mathrm{~W} / \mathrm{cm}^{2}$, there is an increase in the degree of interaction between the radiation and the laser torch, which leads in turn to a decrease in the portion of pulse energy that has reached the target surface [46-48]. The conditions for the formation of the condensed phase of metals subjected to the action of intense pulsed laser radiation of nanosecond length $\left(W \sim 10^{8}-10^{9} \mathrm{~W} / \mathrm{cm}^{2}\right)$ improve with increasing roughness of their surface, which can be explained by the increase in the effective area of radiation-target surface interaction as well as by the presence of a larger number of erosion centers as compared to smooth surfaces. In this case, smoothing of the target surface by a sequence of repeated laser pulses takes place $[25,26]$.

Nanosized particles formed in the erosion torch of metals can be precipitated into liquid media to form colloidal solutions of corresponding metals [29]. Figure 5 presents the data of investigations of particles precipitated from such colloidal solutions on substrates by the methods of scanning electron microscopy (SEM) and transmission electron microscopy (TEM) $[28,30]$. It should be noted that proper treatment of such colloidal solutions permits obtaining metal-polymer composite films (Fig. 5c) [30,31] exhibiting promising optical and magnetic properties.

The surface of functional metals can also act as an implantation medium. In this case, the condensed phase of the laser target material is precipitated in the form of nanosized island and conic structures. As an example, Figure 6a shows the image of the silicon substrate surface obtained with the help of an atomic-force microscope (AFM), on which conic nanostructures from gold were precipitated in air. From the form of the height distribution function of these nanostructures (Fig. 6b) its characteristic value $(\sim 20 \mathrm{~nm})$ can be estimated. It should be noted that this distribution has an asymmetric form similar to the $\gamma$-distribution typical of condensation processes. Figure $6 \mathrm{c}$ shows the characteristic radiation spectrum of a conic nanoobject excited by a narrow-focus electron beam (electron probe), which points to the fact that the formed nanostructures 
consist solely of the target material. Such nanostructures permit, for example, modifying the optical properties of the surface (due to the surface plasmon resonance effect) of silicon photocells with the aim of upgrading their efficiency in the near-IR region [32].

Since the described processes of bulk and surface modification of materials by nanosized metal structures function under normal atmospheric conditions, effective technologies of atmospheric laser-induced evaporation for a wide range of applications can be developed.

Conclusions. We have considered the reasons for the discrepancies between the available experimental and theoretical data on laser erosion of metals by nanosecond laser pulses in air at atmospheric pressure. An attempt has been made to put in order the existing notions about the evolution of the laser torch within the framework of the integral physical picture of the processes confirmed by experiment. Since laser facilities for nanosecond pulse generation have found wide application, understanding the fundamental principles of the process of laser erosion of metals in the air atmosphere will make it possible to improve the technologies of their laser processing, also with the aim of modifying functional materials by metal nanostructures.

The author expresses deep gratitude to Prof. V. K. Goncharov for the discussion of this paper.

\section{NOTATION}

$A$, integral luminescence intensity of the ELT, rel. unit; $E$, quantum energy of characteristic radiation, $\mathrm{keV} ;, h$, distance between the target and the probing radiation, mm; $l$, laser radiation intensity, rel. unit; $L$, total loss factor of the probing radiation in the ELT; $L_{\text {scat }}$, total scattering loss factor of the probing radiation, rel. unit; $L_{\mathrm{abs}}$, absorption loss factor of the probing radiation, rel. unit; $T$, transmissivity of the erosion torch for the probing radiation, rel. unit; $t$, half-amplitude duration of the laser pulse, ns; $U$, relative radiation intensity of a conic nanoobject; $W$, power density of laser radiation, $\mathrm{W} / \mathrm{cm}^{2} ; \lambda$, radiation wavelength, $\mathrm{nm}$. Subscripts: scat, scattering; abs, absorption.

\section{REFERENCES}

1. S. I. Anisimov, Ya. A. Imas, G. S. Romanov, and Yu. V. Khodyko, Effect of High-Power Laser Radiation on Metals [in Russian], Nauka, Moscow (1970).

2. L. I. Mirkin, Physical Principles of Laser Material Processing [in Russian], Izd. MGU, Moscow (1975).

3. N. N. Rykalin, A. A. Uglov, and A. N. Kokora, Laser Material Processing [in Russian], Mashinostroenie, Moscow (1975).

4. A. M. Prokhorov, V. I. Konov, I. Ursu, and I. N. Mikhéilesku, Laser Radiation Interaction with Metals [in Russian], Nauka, Moscow (1988).

5. Yu. P. Raizer, Laser Radiation Effect [in Russian], Mir, Moscow (1974).

6. B. Ya. Lyubov and É. N. Sobol', Effect of Concentrated Energy Fluxes on Materials [in Russian], Nauka, Moscow (1985).

7. J. F. Ready, Effects of High-Power Laser Radiation, Academic Press, New York (1971).

8. E. N. Sobol, Phase Transformations and Ablation in Laser Treated Solids, Wiley, New York (1995).

9. E. Fogarassy and S. Lazare, Laser Ablation of Electronic Materials, North-Holland, Amsterdam (1992).

10. S. I. Anisimov and V. A. Khohlov, Instabilities in Laser-Matter Interaction, CRC Press, Boca Raton, Fla (1995).

11. D. Bäuerle, Laser Processing and Chemistry, Springer, Heidelberg, Berlin (2011).

12. C. Phipps, Laser Ablation and Its Applications, Springer Science+Business Media, New York (2007).

13. S. I. Anisimov, V. V. Zhakhovskii, N. A. Inogamov, et al., Matter expansion and crater formation under the action of an ultrashort laser pulse, Zh. Éksp. Tekh. Fiz., 130, No. 8, 212-227 (2006).

14. V. K. Goncharov, K. V. Kozadaev, and M. V. Pusyrev, The influence of ND laser irradiation parameters on dynamics of metal condensed phase propagating near target, in: M. Sosa and J. Franco (Eds.), Engineering Physics and Mechanics: Analyses, Prediction and Applications, Nova Science Publishers, New York (2009).

15. N. Kumar, S. Dash, A. K. Tyagi, and R. Baldev, Dynamics of plasma expansion in the pulsed laser material interaction, Sadhana (Indian Academy of Science), 25, No. 4, 493-511 (2010).

16. S. I. Anisimov and B. S. Luk'yanchuk, Selected problems of the laser ablation theory, Usp. Fiz. Nauk, 172, No. 3, 301-333 (2002). 
17. N. D. Arnold, B. S. Luk'yanchuk, N. M. Bityurin, and D. Bäuerle, Modeling of nanosecond-laser ablation: Calculations based on a nonstationary averaging technique (spatial moments), in: Proc. SPIE, 3343, 484-504 (1998).

18. B. S. Luk'yanchuk, W. Marine, S. I. Anisimov, and G. A. Simakina, Condensation of vapor and nanoclusters formation within the vapor plume, produced by ns-laser ablation of Si, Ge and C, in: Proc. SPIE, 3618, 434-452 (1999).

19. L. M. Doeswijk, G. Rijnders, and D. H. A. Blank, Pulsed laser deposition: metal versus oxide ablation, Appl. Phys. A, 78, 263-268 (2004).

20. L. Y. Min'ko and Y. A. Chivel, Investigations of the pulsed laser induced destruction of metals and generation of particles, J. Phys. IV, 175-178 (1994).

21. I. A. Bufetov, S. B. Kravtsov, and V. B. Fedorov, Thermodynamic parameters of nanosecond plasma on a solid target in the radiation field of high-power neodymium laser harmonics with a sharp leading edge of the pulse, Kvantovaya Élektron., 23, No. 6, 535-538 (1996).

22. K. S. Gus'kov and S. Yu. Gus'kov, Efficiency of ablation loading and limiting depth of damage of the material irradiated with a high-power laser pulse, Kvantovaya Élektron., 31, No. 4, 305-310 (2001).

23. T. V. Kononenko, V. I. Konov, S. V. Garnov, et al., Comparative investigation of the ablation of materials by femtosecond and pico/nanosecond laser pulses, Kvantovaya Élektron., 28, No. 2, 167-172 (1999).

24. D. Geohegan, A. Puretzky, G. Duscher, and S. Pennycook, Time-resolved imaging of gas-phase nanoparticle synthesis by laser ablation, Appl. Phys. Lett., 72, No 2, 2987-2989 (1998).

25. V. K. Goncharov and K. V. Kozadaev, Formation of the condensed phase of metals under the action of submicrosecond laser pulses, Inzh.-Fiz. Zh., 83, No. 1, 80-84 (2010).

26. V. K. Goncharov, K. V. Kozadaev, and D. V. Shchegrikovich, Formation of the condensed phase of metals under the action of intense nanosecond laser pulses, Inzh.-Fiz. Zh., 86, No. 4, 754-759 (2013).

27. K. V. Kozadaev, Change in the relief of metal targets under the action of submicrosecond high-power-density laser pulses, Perspek. Mater., No. 6, 71-78 (2011).

28. V. K. Goncharov, K. V. Kozadaev, and D. V. Shchegrikovich, Laser synthesis of optical media with silver nanoparticles by nanosecond pulses in air, Opt. Mem. Neur. Networks (Inform. Opt.), 20, No. 4, 255-259 (2011).

29. V. K. Goncharov, K. V. Kozadaev, and D. V. Shchegrikovich, Investigation of noble metal colloidal systems formed by laser synthesis in air, Adv. Opt. Technol., 2012. Article ID 907292. doi: 10.1155/2012/907292.

30. V. K. Goncharov, K. V. Kozadaev, D. I. Shiman, and D. V. Shchegrikovich, Formation and investigation of optical media containing gold nanoparticles, Inzh.-Fiz. Zh., 85, No. 1, 38-42 (2012).

31. V. K. Goncharov, K. V. Kozadaev, and D. I. Shiman, Formation and complex diagnostics of the spectral-morphological parameters of the nanosized phase of silver in a polymer film, Zh. Prikl. Spektrosk., 77, No. 5, 732-736 (2010).

32. K. V. Kozadaev, Laser synthesis of Ag island-shaped nanostructures in air, in: In: Proc. NAP, 2, No 3 (2013). 03 PISERE05.

33. N. Kawahara, J. L. Beduneau, T. Nakayama, E. Tomita, and Y. Ikeda, Spatially, temporally and spectrally resolved measurement of laser-induced plasma in air, Appl. Phys. B, 86, 605-614 (2011).

34. S. M. Klimentov, T. V. Kononenko, P. A. Pivovarov, et al., The role of plasma in the ablation of materials by ultrashort laser pulses, Kvantovaya Élektron., 31, No. 5, 378-382 (2001).

35. S. M. Klimentov, T. V. Kononenko, P. A. Pivovarov, et al., The role of low-threshold breakdown of air in the ablation of materials by short laser pulses, Tr. Inst. Obshsch. Fiz. im. A. M. Prokhorova, 60, 13-29 (2004).

36. V. K. Goncharov, Action of high-energy neodymium laser radiation pulses of different space-time forms on metals, Inzh.-Fiz. Zh., 74, No. 5, 87-97 (2001).

37. E. Yu. Loktionov, A. V. Ovchinnikov, Yu. Yu. Protasov, and D. S. Sitnikov, Energy efficiency of femtosecond laser ablation of refractory metals, Zh. Prikl. Spektrosk., 77, No. 4, 604-611 (2010).

38. E. Y. Loktionov, A. V. Ovchinnicov, Y. Y. Protasov, and D. S. Sitnicov, Experimental investigation on spectral-energy efficiency of femtosecond laser ablation of metals, Plasma Phys. Rep., 37, No. 13, 1208-1214 (2011).

39. J. Byskov-Nielsen, J.-M. Savolainen, M. S. Christensen, and P. Balling, Ultra-short pulse laser ablation of copper, silver and tungsten: experimental data and two-temperature model simulations, Appl. Phys. A, 103, 447-453 (2011).

40. I. Mingareev and A. Horn, Time-resolved investigations of plasma and melt ejections of metals by pump-probe shadowgraphy, Appl. Phys. A, 92, 917-920 (2008).

41. I. K. Kikoin, Tables of Physical Quantities [in Russian], Atomizdat, Moscow (1976).

42. A. Evtushenko, E. Ivanik, and K. Rozhnyakovskii, On one method for determining the effective absorption coefficient under pulsed laser irradiation of metals, Inzh.-Fiz. Zh., 76, No. 5, 10-15 (2003). 
43. A. F. Banishev and E. A. Balykina, Damage of the silicon and copper surface under pulsed and pulsed-periodic YAG-Nd laser irradiation, Kvantovaya Élektron., 24, No. 6, 557-559 (1997).

44. S. I. Anisimov, V. A. Gal'burt, M. F. Ivanov, et al., On the theory of laser interaction with metals, Zh. Tekh. Fiz., 49, No. 3, 512-517 (1979).

45. V. K. Goncharov, K. V. Kozadaev, V. V. Makarov, and D. V. Shchegrikovich, Proceeding of erosion processes in the near-surface region of metals irradiated with intense nanosecond laser pulses, Inzh.-Fiz. Zh., 86, No. 4, 747-753 (2013).

46. V. K. Goncharov, K. V. Kozadaev, and D. V. Shchegrikovich, Onset of condensation in erosion torches of metals irradiated with high-intensity submicrosecond laser pulses, Inzh.-Fiz. Zh., 84, No. 4, 723-728 (2011).

47. S. I. Anisimov, N. A. Inogamov, Y. V. Petrov, and V. A. Khohlov, Interaction of short laser pulses with metals at moderate intensities, Appl. Phys. A, 92, 939-943 (2008).

48. V. K. Goncharov, K. V. Kozadaev, and D. V. Shchegrikovich, Dynamics of the optical characteristics of erosion laser torches of metals irradiated with intense nanosecond pulses under atmospheric conditions, Zh. Prikl. Spektrosk., 80, No. 3, 409-416 (2013).

49. L. T. Sukhov, Laser Spectral Analysis [in Russian], Nauka, Novosibirsk (1990).

50. J. Singh and S. Thakur, Laser-Induced Breakdown Spectroscopy, Elsevier, Amsterdam (2007).

51. Ya. B. Zel'dovich and Yu. P. Raizer, Physics of Shock Waves and High-Temperature Hydrodynamic Phenomena [in Russian], Nauka, Moscow (1966).

52. A. N. Chumakov, V. V. Efremov, N. A. Bosak, et al., Radiation of laser microplasma formations in the visible and IR spectral regions, Kvantovaya Élektron., 21, No. 8, 773-777 (1994).

53. M. P. Chuchman and A. K. Shuaibov, Emission characteristics and properties of a laser-plume germanium plasma, Plasma Phys. Rep., 34, No. 4, 306-311 (2008).

54. V. K. Unnikrishnan, A. Kamlesh, V. B. Kartha, et al., Measurements of plasma temperature and electron density in laserinduced copper plasma by time-resolved spectroscopy of neutral atom and ion emissions, Pramana J. Phys., 74, No. 6, 983-993 (2010).

55. M. Kubkowska, P. Gasior, M. Rosinski, et al., Characterization of laser-produced tungsten plasma using optical spectroscopy method, Eur. Phys. J., 54, 463-466 (2009).

56. A. K. Shuaibov, M. P. Chuchman, and L. L. Shimon, Spectroscopic diagnostics of the laser erosion plasma of lead, Tech. Phys. Lett., 30, No. 12, 1042-1044 (2004).

57. M. Kuwata, B. Luk'yanchuk, and T. Yabe, Nanoclusters formation within the vapor plume, produced by ns-laser ablation: Effect of the initial density and pressure distributions, in: Proc. SPIE, 4065, 441-451 (2000). 